\title{
THE SINS SURVEY: BROAD EMISSION LINES IN HIGH-REDSHIFT STAR-FORMING GALAXIES*
}

\author{
Kristen L. Shapiro ${ }^{1}$, Reinhard Genzel ${ }^{2,3}$, Eliot Quataert ${ }^{1}$, Natascha M. Förster Schreiber $^{3}$, Richard Davies $^{3}$, \\ Linda TacConi $^{3}$, Lee Armus ${ }^{4}$, Nicolas Bouché ${ }^{3}$, Peter Buschkamp ${ }^{3}$, Andrea Cimatti ${ }^{5}$, Giovanni Cresci $^{3}$, \\ Emanuele Daddi ${ }^{6}$, Frank Eisenhauer ${ }^{3}$, Dawn K. Erb ${ }^{7}$, Shy Genel ${ }^{3}$, Erin K. S. Hicks ${ }^{3}$, Simon J. Lilly ${ }^{8}$, Dieter Lutz ${ }^{3}$, \\ Alvio Renzini ${ }^{9}$, Alice Shapley ${ }^{10}$, Charles C. Steidel $^{11}$, and Amiel Sternberg ${ }^{12}$ \\ ${ }^{1}$ Department of Astronomy, University of California, Berkeley, CA 94720, USA \\ ${ }^{2}$ Department of Physics, University of California, Berkeley, CA 94720, USA \\ ${ }^{3}$ Max-Planck-Institut für extraterrestrische Physik (MPE), Giessenbachstr.1, D-85748 Garching, Germany \\ ${ }^{4}$ Spitzer Science Center, California Institute of Technology, Pasadena, CA 91125, USA \\ ${ }^{5}$ Istituto Nazionale di Astrofisica-Osservatorio Astronomico di Bologna, Via Gobetti 101, I-40129 Bologna, Italy \\ ${ }^{6}$ Service d'Astrophysique, Dapnia CEA, Saclay, France \\ ${ }^{7}$ Harvard-Smithsonian Center for Astrophysics, 60 Garden Street, Cambridge, MA 02138, USA \\ ${ }^{8}$ Institute of Astronomy, Eidgenössische Technische Hochschule, ETH Zurich CH-8093, Switzerland \\ ${ }^{9}$ Osservatorio Astronomico di Padova, Vicolo dell'Osservatorio 5, Padova I-35122, Italy \\ ${ }^{10}$ Department of Physics and Astronomy, University of California, Los Angeles, CA 90095, USA \\ ${ }^{11}$ California Institute of Technology, MS 105-24, Pasadena, CA 91125, USA \\ ${ }^{12}$ School of Physics and Astronomy, Tel Aviv University, Tel Aviv 69978, Israel \\ Received 2009 February 11; accepted 2009 June 16; published 2009 July 28
}

\begin{abstract}
High signal-to-noise, representative spectra of star-forming galaxies at $z \sim 2$, obtained via stacking, reveal a high-velocity component underneath the narrow $\mathrm{H} \alpha$ and [N II] emission lines. When modeled as a single Gaussian, this broad component has FWHM $\gtrsim 1500 \mathrm{~km} \mathrm{~s}^{-1}$; when modeled as broad wings on the $\mathrm{H} \alpha$ and [N II] features, it has FWHM $\gtrsim 500 \mathrm{~km} \mathrm{~s}^{-1}$. This feature is preferentially found in the more massive and more rapidly star-forming systems, which also tend to be older and larger galaxies. We interpret this emission as evidence of either powerful starburst-driven galactic winds or active supermassive black holes (SMBHs). If galactic winds are responsible for the broad emission, the observed luminosity and velocity of this gas imply mass outflow rates comparable to the star formation rate. On the other hand, if the broad-line regions of active black holes account for the broad feature, the corresponding black holes masses are estimated to be an order of magnitude lower than those predicted by local scaling relations, suggesting a delayed assembly of SMBHs with respect to their host bulges.
\end{abstract}

Key words: galaxies: active - galaxies: evolution - galaxies: high-redshift

\section{INTRODUCTION}

Observations of galaxies in the early universe are a unique probe of matter assembly during its most active epoch; at $z \sim 2$, both the cosmic star formation rate and the luminous quasar space density are at their peaks (e.g., Fan et al. 2001; Chapman et al. 2005). Galaxies themselves undergo corresponding growth during this time, with the total stellar mass density in galaxies increasing from $\sim 15 \%$ to $50 \%-75 \%$ its current value between $z \sim 3$ and $z \sim 1$ (e.g., Dickinson et al. 2003; Rudnick et al. 2003, 2006). Constraining the dynamical and baryonic processes driving this rapid evolution is, therefore, central to our understanding of galaxy formation and to informing cosmological simulations.

At the relevant epochs, key spectral diagnostic features are redshifted into the near-infrared. In recent years, a number of surveys have therefore begun to systematically probe highredshift populations with near-infrared spectroscopy (e.g., Erb et al. 2006a, 2006b, 2006c; Swinbank et al. 2004; Takata et al. 2006; Kriek et al. 2008). Our recently completed SINS (Spectroscopic Imaging in the Near-infrared with SINFONI) survey has combined the resolving power of $8-10 \mathrm{~m}$ class telescopes with high-resolution integral field spectrographs to

\footnotetext{
* Based on observations obtained at the Very Large Telescope (VLT) of the European Southern Observatory, Paranal, Chile in the context of ESO programs 070.A-0229, 070.B-0545, 073.B-9018, 074.A-9011, 075.A-0466, 076.A-0527, 077.A-0576, 078.A-0055, 078.A-0600, 079.A-0341, 080.A-0330, and $080 . \mathrm{A}-0635$.
}

study the detailed internal processes at work within massive, star-forming galaxies at $z \sim 2$ (Förster Schreiber et al. 2006, 2009; Genzel et al. 2006, 2008; Bouché et al. 2007; Shapiro et al. 2008; Cresci et al. 2009; see also related work by Puech et al. 2006; Swinbank et al. 2006; Wright et al. 2007; Law et al. 2007, 2009).

In this paper, we combine the spectra of 47 galaxies detected in $\mathrm{H} \alpha$ emission to study the average spectral properties of starforming galaxies at $z \sim 2$ (Section 2). In particular, we report the discovery of broad emission lines in these galaxies (Section 3) and interpret this high-velocity warm gas as arising either in large-scale galactic winds driven by the high star formation rates (SFRs) in these galaxies or in the broad-line regions (BLRs) surrounding active galactic nuclei (AGNs). We explore the implications of both scenarios in Section 4 and conclude in Section 5.

Throughout this paper, we assume a $\Lambda$-dominated cosmology with $H_{0}=70 \mathrm{~km} \mathrm{~s}^{-1} \mathrm{Mpc}^{-1}, \Omega_{\mathrm{m}}=0.3$, and $\Omega_{\Lambda}=0.7$. For this cosmology, $1^{\prime \prime}$ corresponds to $\approx 8.2 \mathrm{kpc}$ at $z=2.2$.

\section{DATA AND ANALYSIS}

In the context of the SINS program, $80 z=1-3$ systems were observed in emission lines in the infrared (rest-frame optical) with VLT/SINFONI (Eisenhauer et al. 2003; Bonnet et al. 2004) for an average of $3.5 \mathrm{hr}$ per band and pixel scale on each target (Förster Schreiber et al. 2009). These galaxies were largely $(62 / 80)$ taken from the (rest-frame) UV-selected samples of Erb et al. (2006b, 2006c) and the (rest-frame) optically-selected 

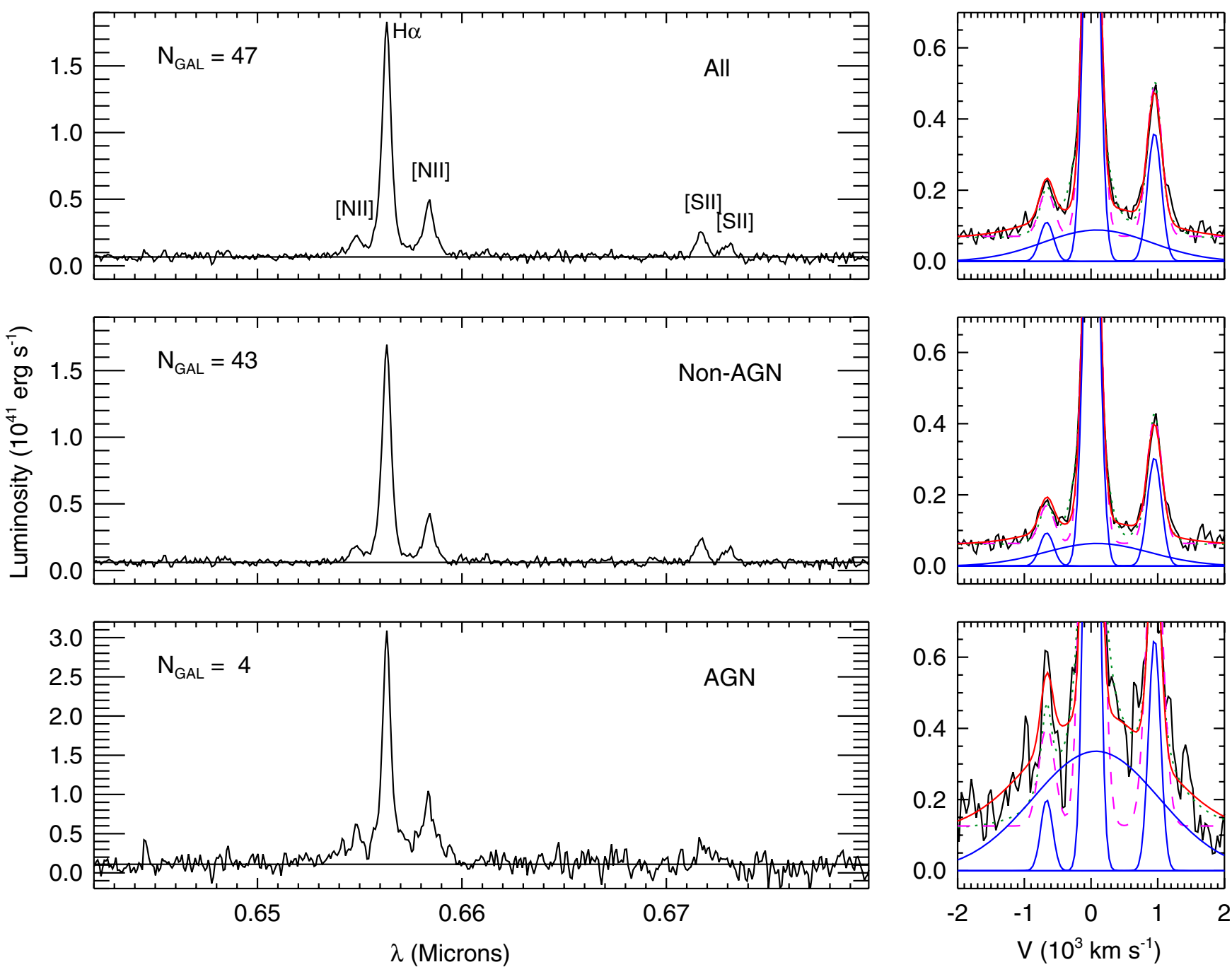

Figure 1. Left: average spectrum of the 47 SINS galaxies (top panel), followed by the average spectrum of all nonactive systems and that of all systems previously known to host AGNs (lower two panels). Right: zoomed view of the $\mathrm{H} \alpha$ and [N II] region, on a velocity scale, with the best-fitting combination of a constant continuum, narrow lines, and a broad component overplotted (red). The individual line components are also plotted (blue). For reference, also overplotted are the best fits derived by fitting both $\mathrm{H} \alpha$ and [N II] with only narrow lines (dashed magenta) and with a narrow and a broad component (dotted green).

samples of Abraham et al. (2004), Daddi et al. (2004b), Kong et al. (2006), Lilly et al. (2007), and J. Kurk et al. (2010, in preparation). From these magnitude- and color-defined samples, suitable SINS targets were culled, with the main selection criteria being a combination of target visibility during the observing runs, night sky line avoidance in the emission lines of interest, and an estimated integrated emission-line flux $\gtrsim 5 \times 10^{-17} \mathrm{erg} \mathrm{s}^{-1} \mathrm{~cm}^{-2}$, such that high-quality data could be obtained in reasonable integration times. Of the 62 rest-frame $\mathrm{UV} /$ optically-selected galaxies chosen in this manner, 52 were well detected in $\mathrm{H} \alpha$ in our SINFONI observations.

Förster Schreiber et al. (2009) discuss the selection of this sample in detail and show that the SINS galaxies are representative of the $z \sim 2$ star-forming galaxy population, with some bias toward the more rapidly star-forming (and therefore more luminous in $\mathrm{H} \alpha$ emission) systems. They also note that the SINS sample (and some of the parent samples) selects against known AGNs and quasars, in the interest of studying the dynamic and evolutionary state of $z \sim 2$ starforming galaxies. However, a small number of previously known AGNs were in fact observed in the SINS program; in the UV/ optically-selected part of the sample, there are five such systems, as originally identified with the UV/optical spectroscopy of the parent surveys. For further details about the SINS sample, observations, and data reduction, we refer interested readers to Förster Schreiber et al. (2009).

Here, we analyze our SINS observations of the galaxies that were UV/optically identified and that are well detected in $\mathrm{H} \alpha$ in individual spatial elements; this population (totalling 47 of 52 sources detected in $\mathrm{H} \alpha$, including $4 / 5$ of the previously known AGNs) comprises the majority of the SINS sample. To study the average properties of these $z \sim 2$ star-forming galaxies, we generated stacked, high-signal-to-noise $(\mathrm{S} / \mathrm{N})$ spectra representative of the population as a whole.

From our integral field data, we first created a spatially integrated one-dimensional spectrum for each galaxy by shifting each spectrum within the galaxy datacube by its measured $(\mathrm{H} \alpha)$ velocity and then collapsing the datacube into a single spatially integrated spectrum. In this manner, the spatially integrated spectrum contains no systematic velocity broadening (e.g., by large-scale rotation) on scales larger than the pointspread function (PSF; FWHM $\sim 4 \mathrm{kpc}$ ). Testing confirmed that this approach did not affect the properties of the broad $\mathrm{H} \alpha$ component (see below) but did improve the $\mathrm{S} / \mathrm{N}$ of the detection. This technique has the additional benefit of randomizing $\mathrm{OH}$ atmospheric emission lines in the $\mathrm{H} \alpha$ rest frame and therefore 
Table 1

Results from Line Fitting

\begin{tabular}{|c|c|c|c|c|c|c|c|c|}
\hline Subsample & $\begin{array}{c}\text { Figure } \\
\text { Reference }\end{array}$ & $\begin{array}{c}L_{\mathrm{H} \alpha, \text { broad }} \\
\left(10^{41} \mathrm{erg} \mathrm{s}^{-1}\right)\end{array}$ & $f_{\text {broad }^{a}}{ }^{\mathrm{a}}$ & $\begin{array}{c}v_{\text {off }^{\mathrm{b}}} \\
\left(\mathrm{km} \mathrm{s}^{-1}\right)\end{array}$ & $\begin{array}{c}\text { FWHM }_{\mathrm{H} \alpha \text {,broad }} \\
\left(\mathrm{km} \mathrm{s}^{-1}\right)\end{array}$ & $P_{\text {false }}$ & $\chi_{\mathrm{dof}}^{2}$ & $\chi_{\text {dof,narrow }}^{2}$ \\
\hline All & 1 , top & $4.0_{-1.0}^{+1.1}$ & $0.28_{-0.08}^{+0.04}$ & $18_{-73}^{+79}$ & $1632_{-301}^{+445}$ & 0.02 & 1.8 & 4.9 \\
\hline All, broad $\mathrm{H} \alpha$, and $\left[\mathrm{N}_{\mathrm{II}}\right]^{\mathrm{d}}$ & 1 , top & $5.9_{-1.4}^{+1.0}$ & $0.42_{-0.08}^{+0.08}$ & $8_{-3}^{+14}$ & $556_{-87}^{+228}$ & 0.01 & 1.4 & 4.9 \\
\hline Non-AGN & 1 , center & $3.0_{-0.4}^{+0.6}$ & $0.22_{-0.02}^{+0.03}$ & $19_{-42}^{+82}$ & $1329_{-139}^{+346}$ & 0.03 & 1.9 & 3.7 \\
\hline AGN & 1 , bottom & $8.2_{-2.8}^{+0.7^{4}}$ & $0.36_{-0.09}^{+0.03}$ & $42_{-29}^{+94}$ & $2863_{-728}^{+669}$ & 0.007 & 0.9 & 3.0 \\
\hline$M_{*}<2 \times 10^{10} M_{\odot}$ & 2, top & $2.2_{-0.6}^{+0.4}$ & $0.21_{-0.02}^{+0.03}$ & $26_{-30}^{+86}$ & $1051_{-169}^{+149}$ & 0.11 & 0.9 & 1.3 \\
\hline$M_{*}=2-7 \times 10^{10} M_{\odot}$ & 2 , center & $2.9_{-0.7}^{+0.6}$ & $0.22_{-0.02}^{+0.06}$ & $11_{-70}^{+71}$ & $1425_{-238}^{+443}$ & 0.05 & 1.4 & 2.0 \\
\hline$M_{*}>7 \times 10^{10} M_{\odot}$ & 2 , bottom & $6.7_{-2.3}^{+2.9}$ & $0.31_{-0.11}^{+0.04}$ & $20_{-42}^{+82}$ & $2183_{-758}^{+392}$ & 0.03 & 1.3 & 3.6 \\
\hline Center & 3 , top & $5.1_{-1 .}^{+2.8 \mathrm{e}}$ & $0.24_{-0.04}^{+0.13}$ & $9_{-27}^{+80}$ & $1564_{-544}^{+601}$ & 0.08 & 1.3 & 1.9 \\
\hline Extended & 3 , bottom & $1.9_{-0.5}^{+0.6 \mathrm{e}}$ & $0.22_{-0.02}^{+0.06}$ & $10_{-39}^{+69}$ & $1508_{-244}^{+725}$ & 0.14 & 0.9 & 1.2 \\
\hline
\end{tabular}

Notes.

a Ratio of $\mathrm{H} \alpha$ luminosity in broad component to total $\mathrm{H} \alpha$ (narrow+broad) luminosity.

b Velocity offset of broad $\mathrm{H} \alpha$ feature from narrow $\mathrm{H} \alpha$.

c Reduced $\chi^{2}$ for a fit assuming only narrow emission lines.

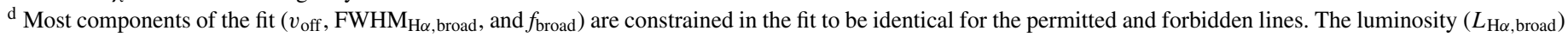
is quoted only for the broad component of the $\mathrm{H} \alpha$ line.

${ }^{\mathrm{e}}$ Units are $10^{39} \mathrm{erg} \mathrm{s}^{-1} \mathrm{kpc}^{-2}$.

effectively eliminating residuals from the $\mathrm{OH}$ line removal. The remaining residuals from this process were inspected and masked out by hand.

The 47 spatially integrated spectra were then combined into a single spectrum (with equivalent integration time of $195 \mathrm{hr}$ ) by interpolating all spectra onto a common wavelength axis, converting their measured fluxes to luminosities using their luminosity distances, weighting each spectrum by the $\mathrm{S} / \mathrm{N}$ of the $\mathrm{H} \alpha$ emission line, and averaging the resulting spectra. During this process, we do not correct for extinction (but see Section 4). Typical extinctions of our sample galaxies have been measured to be $A_{V} \sim 1$ (Förster Schreiber et al. 2009; see also, e.g., Daddi et al. 2004a; Erb et al. 2006c), which translates to an underestimation of our $\mathrm{H} \alpha$ luminosities by at most a factor of $\sim 2$.

The average spectrum for the SINS $z \sim 2$ star-forming galaxies is presented in the top panel of Figure 1. We also created average spectra of subsets of the SINS galaxy sample, in order to test the dependence of spectral properties on other known galaxy properties.

Our average spectra reveal a broad emission component underneath the bright narrow lines. We quantify this feature in each average spectrum by simultaneously fitting a combination of a constant continuum offset, narrow lines ( $\mathrm{H} \alpha,[\mathrm{N} \mathrm{II}],[\mathrm{S} \mathrm{II}])$ of identical kinematics (velocity and velocity dispersion), and a single broad component, whose kinematics are allowed to vary. All lines are assumed to be well described by a single Gaussian; the validity of this assumption is confirmed by a reduced $\chi^{2}$ close to unity $\left(\chi_{\text {dof }}^{2} \sim 0.9-1.9\right)$ for all fits (see Table 1). Our data can also be well fitted by a combination of a constant continuum offset, narrow lines with shared kinematics, and broad forbidden and permitted lines with shared kinematics, with the $[\mathrm{N} \mathrm{II}] / \mathrm{H} \alpha$ ratio identical in the narrow and broad components. All lines are assumed to be well described by this combination of two Gaussians (dotted green line in Figure $1 ; \chi_{\text {dof }}^{2}=1.4$ ), which has the same number of free parameters as the previous fit. In the limit of the $\mathrm{S} / \mathrm{N}$ of our data, we cannot add additional free parameters to the fits, nor can we identify a preferred model. For clarity, we refer throughout to the former (single broad Gaussian under the $\mathrm{H} \alpha+\left[\mathrm{N}_{\text {II }}\right]$ complex) as "broad lines" and the latter (double Gaussians for both $\mathrm{H} \alpha$ and [N $\mathrm{NI}_{\mathrm{II}}$ ) as "broad wings." For simplicity and for comparison with the literature, we primarily quantify the observed high-velocity feature with a single broad $\mathrm{H} \alpha$ line in Section 3. However, we also discuss the implications of the broad wings scenario on the derived properties of the broad emission (Section 3) and on the interpretation of this emission (Section 4).

From these fits to the average spectra, we measure the fractional contribution of broad emission to the overall emissionline flux, the kinematics of the broad component, and the line ratios of the narrow-line components. The significance of these measurements is quantified in two ways. First, for each average spectrum, we recreated 100 spectra by randomly sampling (with replacement) and combining the individual contributing galaxy spectra. The properties of the broad component were measured in each of the resulting 100 average spectra, yielding confidence intervals for all derived quantities. Second, the probability of false positive detections $P_{\text {false }}$ was tested by creating 1000 simulated spectra with the narrow line and observational properties (noise and spectral resolution) characteristic of each average spectrum. Comparing the derived broad components in these spectra to those in the real SINS spectra, we estimate the rate of spurious detections of broad components equal to or more prominent (in luminosity and FWHM) than those in the actual data. The results of this analysis for the different average spectra are presented in the following section.

\section{RESULTS}

We find that the average SINS galaxy spectrum includes a significant amount of broad emission (top panel of Figure 1), with $\chi_{\text {dof }}^{2}=4.9$ for a fit with only narrow lines (dashed magenta line in the top right panel of Figure 1) and $\chi_{\text {dof }}^{2}=1.8$ and 1.4 for fits including a broad $\mathrm{H} \alpha$ line and broad $\mathrm{H} \alpha$ and [N II] wings, respectively (red and dotted green lines in the same panel; $P_{\text {false }}=2 \%$ and $1 \%$ ). To ensure that this signature is not the result of the four known AGNs included in this sample, we also create stacked spectra of the AGNs and the rest of the sample. While the broad emission from the AGN host systems alone is quite substantial, a comparison of the average SINS spectrum and the average non-AGN spectrum illustrates that the broad emission in the average SINS spectrum is not dominated by that coming from the four AGNs. These results are summarized in Table 1. 

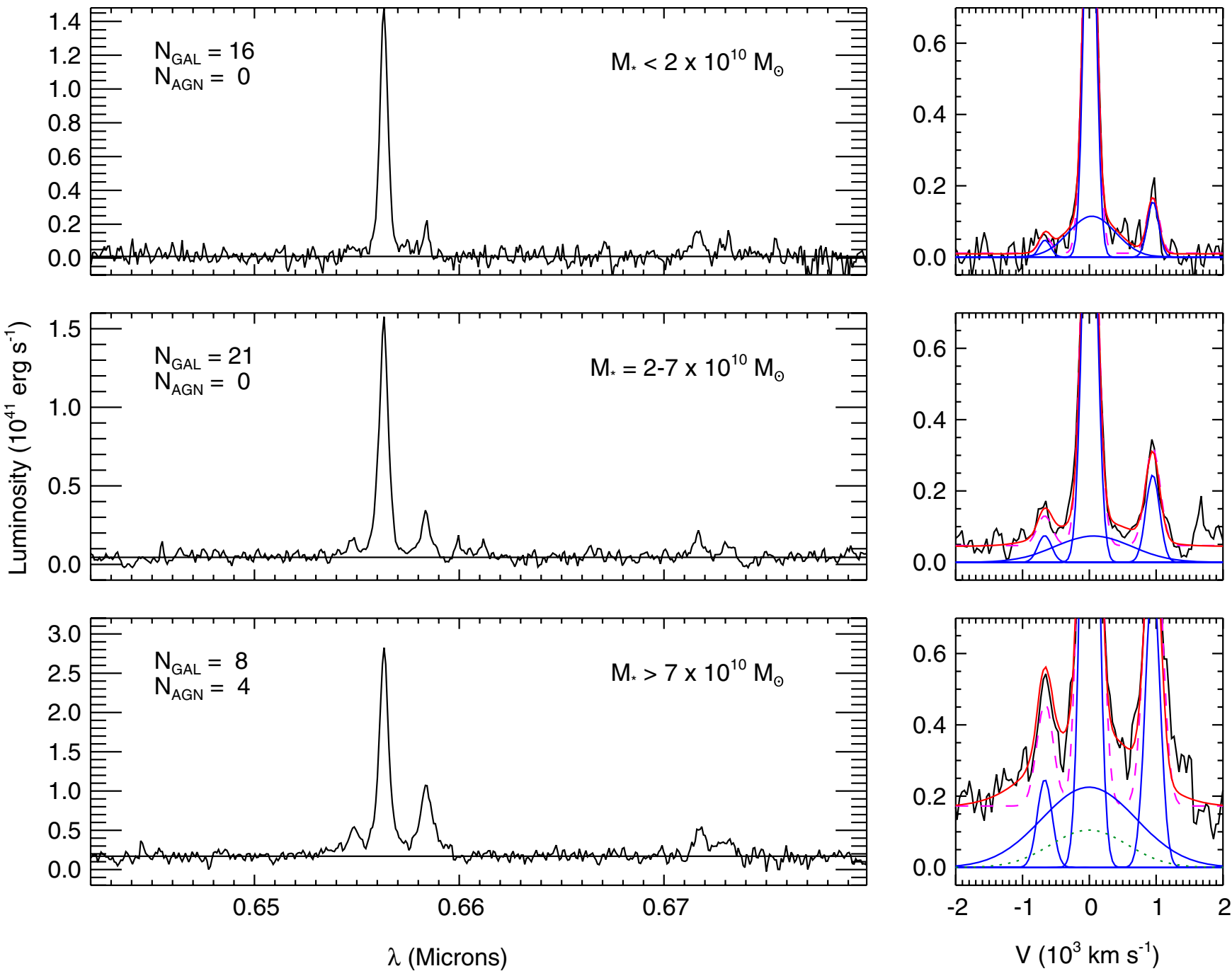

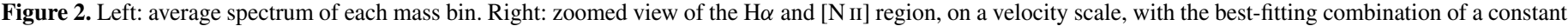

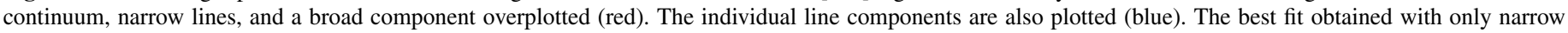

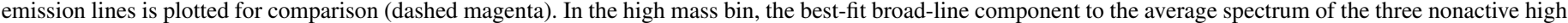
mass galaxies is also overplotted (dotted green).

We test the dependence of the presence of broad emission on galaxy properties by dividing the sample into three stellar mass bins, using the results from the spectral energy distribution (SED) fitting of Förster Schreiber et al. (2009) for the 45 of our 47 targets for which sufficient broadband data exist. The average spectra for these three bins show an increasing presence of a broad component with stellar mass (Figure $2 ; P_{\text {false }}=11 \%, 5 \%$, and $3 \%$, respectively). However, the spectrum of the highest mass bin is significantly affected by the contribution of the four previously known AGNs in our sample, which all fall into this bin. The nuclear emission in these systems can bias the results of SED fitting toward larger masses, so we confirm their high masses with the dynamical mass measurements made by Cresci et al. (2009); in all cases, the dynamical masses of these galaxies are consistent with the stellar masses used here and remain among the highest in the SINS sample. Nevertheless, we confirm that a (weaker) broad component is also present in the nonactive galaxies in this bin (green line in Figure 2), with $P_{\text {false }}=9 \%$.

Several other key properties of galaxies, including SFR, size, stellar age, and metallicity, have well established correlations with stellar mass in high-z galaxies (e.g., Noeske et al. 2007;
Trujillo et al. 2006; Erb et al. 2006b). Both the SFR $-M_{*}$ relation and the mass-metallicity relation are apparent in Figure 2, via the increasing narrow $\mathrm{H} \alpha$ luminosity and increasing [N $\mathrm{NI}] / \mathrm{H} \alpha$ ratio with stellar mass, respectively. Since the $\left[\mathrm{N}_{\mathrm{II}}\right] / \mathrm{H} \alpha$ ratio remains well below levels expected of shock heating or AGN activity, this latter is most likely tracing variations in metallicity (see below, as well as P. Buschkamp et al. 2010, in preparation). To these established mass-dependent properties in high- $z$ galaxies, we now add the presence and strength of a broad component.

With the spatially resolved data, we can also compare the integrated spectra from the central $(R<3 \mathrm{kpc})$ regions of high$z$ galaxies to those from extended $(R=3-15 \mathrm{kpc})$ regions, in order to determine which regions in these systems are generating broad emission. For this analysis, we use galaxies from the intermediate and high mass bins of Figure 2 in which the intensity distribution of the stellar continuum defines a clear center of the system (totalling six systems). The average spectra of the central and extended regions of these systems are shown in Figure 3, normalized to the spatial area over which the spectra were extracted.

In these spectra, a broad component is preferred by the bestfitting models; however, the significance of this result is low. 

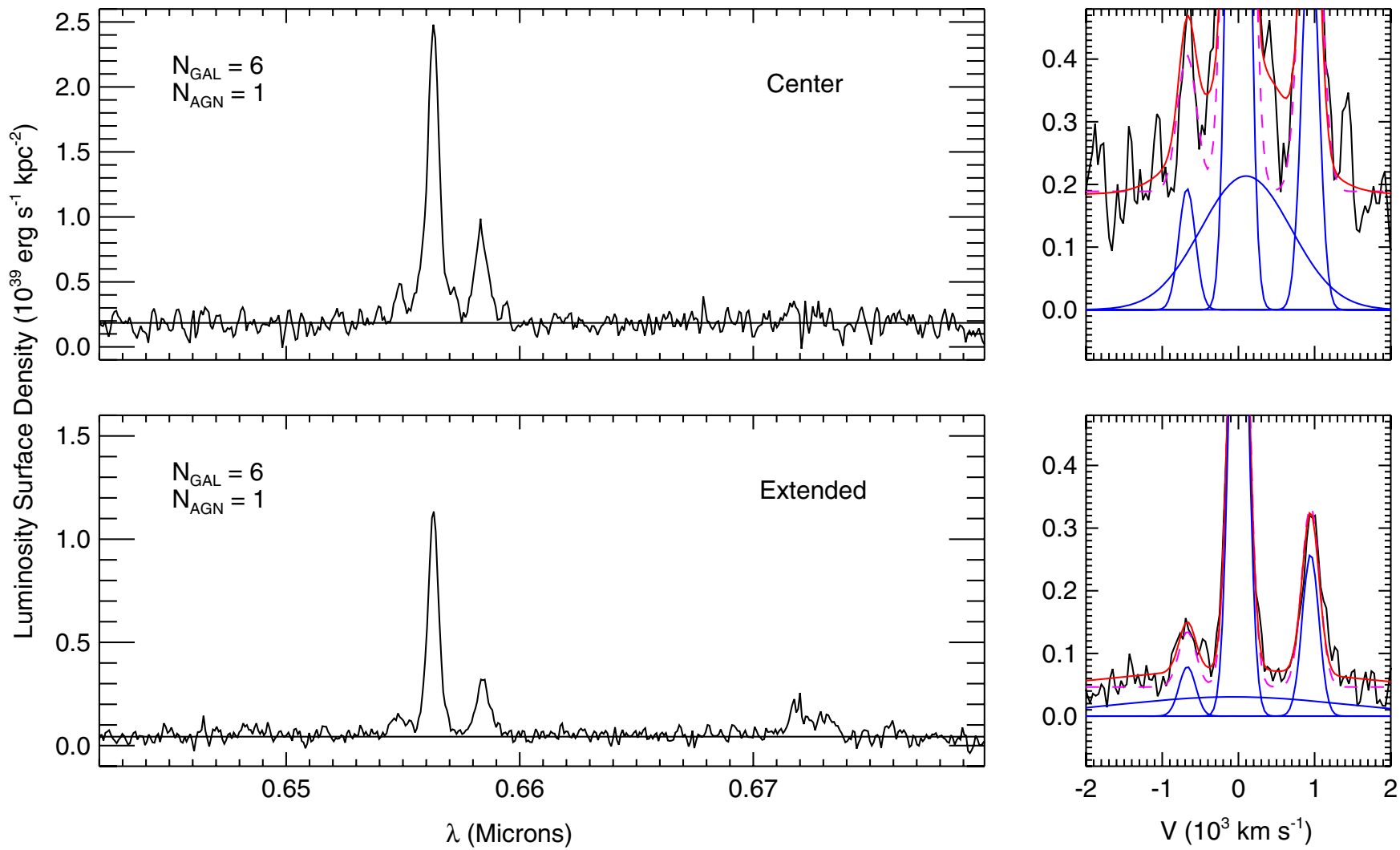

Figure 3. Average spectra, in luminosity surface density, of the central (top panel) and extended (bottom panel) regions of well resolved SINS galaxies. Panels and colors are as in Figures 1 and 2.

The detection of broad emission in galaxy centers at $z \sim 2$ (with $\left.P_{\text {false }}=8 \%\right)$ is somewhat more robust than that in the extended regions $\left(P_{\text {false }}=14 \%\right)$, in which the best-fit broad component is very shallow. If real, the broad feature in the extended regions accounts for a comparable fraction of the total $\mathrm{H} \alpha$ luminosity to that in the central regions (Table 1). Tests of simulated galaxies indicate that such a broad line in the extended regions cannot be reproduced by a nuclear point source of broad emission (i.e., AGN) broadened by the PSF.

In the spectra shown in Figure 3, we note that the average $[\mathrm{N} I I] / \mathrm{H} \alpha$ ratio is comparable in the central and extended regions, despite the difference in broad emission component, in support of the above interpretation of the $\left[\mathrm{N}_{\mathrm{II}}\right] / \mathrm{H} \alpha$ feature as primarily reflecting the metallicity of these systems. However, this should not be interpreted as a lack of metallicity gradient in these galaxies, since the spectra shown here are averaged over a number of galaxies, in each of which the extended region is spatially integrated over a large range in radii. Detailed studies of metallicity gradients within and between individual SINS systems will be presented in P. Buschkamp et al. (2010, in preparation).

\section{DISCUSSION}

The low-luminosity broad emission seen in our SINS galaxies can be interpreted in one of two ways: either as evidence of large-scale galactic outflows, presumably driven by the galaxies' very high SFRs, or as a tracer of the BLRs surrounding AGNs in these early galaxies. The current data do not allow us to robustly distinguish between these two scenarios empirically; in the following, we therefore examine both in detail.

\subsection{Broad Emission from Starburst-driven Winds}

One possible explanation of the broad emission is a starburstdriven wind. This scenario is in keeping with the positive correlation between broad emission and stellar mass (and therefore SFR) seen in Figure 2 and with the possible broad emission from non-nuclear regions seen in Figure 3. Moreover, starburst-driven winds are expected to be ubiquitous in the rapidly star-forming populations common at high redshift (e.g., Pettini et al. 2001; Shapley et al. 2003; Smail et al. 2003; Weiner et al. 2009) and are probably expelling mass from their host galaxies at rates comparable to the SFR (below; see also Martin 1999; Pettini et al. 2000; Martin 2003; Erb 2008; Weiner et al. 2009).

At low redshift, star-forming systems are known to drive galactic winds with observable signatures in the wings of the permitted and forbidden emission lines. In dwarf starburst galaxies, Westmoquette et al. (2007a) find that the broad wings of the emission lines can be modeled as a second Gaussian component with FWHM $\leqslant 300 \mathrm{~km} \mathrm{~s}^{-1}$. In contrast, observations of the more massive and more rapidly star-forming IR-luminous galaxy population reveal higher FWHM in the broad wings (300-800 $\mathrm{km} \mathrm{s}^{-1}$; Arribas et al. 2001), whose line widths and $f_{\text {broad }}$ are comparable to those observed in the SINS galaxies (550 $\mathrm{km} \mathrm{s}^{-1}$; see Table 1; Armus et al. 1989, 1990; Lehnert \& Heckman 1996).

Indeed, the relationship between wind speed and SFR (and the equivalent properties, galaxy mass and $B$-band magnitude) has been demonstrated by Rupke et al. (2005), Martin (2005), and Tremonti et al. (2007), respectively. In particular, Lehnert \& Heckman (1996) and Rupke et al. (2005) showed that systems with higher SFR have faster winds, whose velocities exceed 


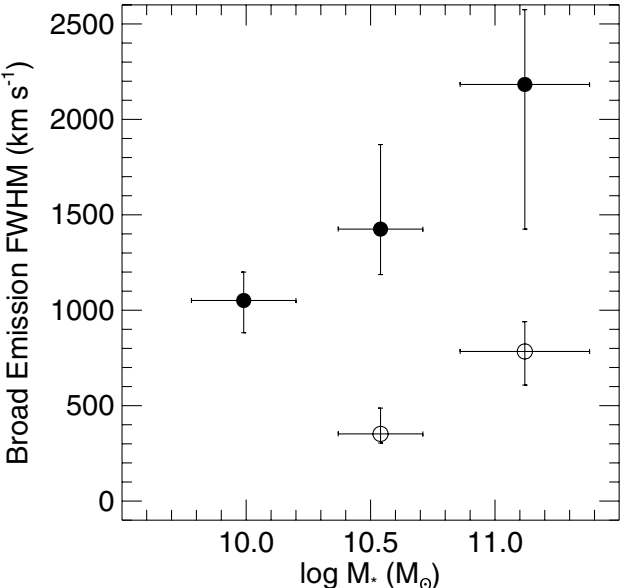

Figure 4. FWHM of the broad component in the SINS galaxies in the three stellar mass bins defined in Figure 2, as modeled by a single broad $\mathrm{H} \alpha$ line (filled circles) and by broad wings on both the $\mathrm{H} \alpha$ and [N II] lines (open circles). In the latter model, the low mass bin is not well constrained due to the low $\mathrm{S} / \mathrm{N}$ of the [N II] feature (see Figure 2), resulting in a fit that strongly prefers a broad line for only the high $\mathrm{S} / \mathrm{N}(\mathrm{H} \alpha)$ line; we therefore omit this bin from the plot.

$\sim 1000 \mathrm{~km} \mathrm{~s}^{-1}$ in ionized gas tracers. We recover similar trends and velocities with the three mass bins of the SINS data, as shown in Figure 4. Note, however, that this figure should not be directly compared with those in the works listed above, in which the wind speeds are probed with interstellar absorption features, whose velocities are typically lower than those of ionized emission-line gas (e.g., Veilleux et al. 2005; Rupke et al. 2005). Nevertheless, the similarity between the properties of local winds and those of the broad wings seen in the SINS galaxies makes it plausible that this emission is due to the galactic winds that certainly exist in these systems.

These broad wings can be produced via several mechanisms, including turbulent mixing of the hot wind fluid and cool gas clumps at the base of the wind (Westmoquette et al. 2007a, $2007 \mathrm{~b}$ ) or within the large-scale bubble along the galaxy minor axis (e.g., Armus et al. 1990; Arribas et al. 2001). In the idealized model of a wind plowing into a spherically symmetric ambient medium, Heckman et al. (1993) provide analytic expressions for the velocity and $\mathrm{H} \alpha$ luminosity of the shocked gas as functions of the bolometric luminosity of the star-formation event, the duration of the event, and the ISM density. Converting the SFR $\left(\sim 100 M_{\odot} \mathrm{yr}^{-1}\right)$ of the SINS galaxies to a bolometric luminosity and accounting for the correspondingly denser ISM than in local spiral galaxies (by a factor of 10-30; Bouché et al. $2007)$, these relations predict a $\mathrm{H} \alpha$ luminosity of $(0.7-10) \times$ $10^{42} \mathrm{erg} \mathrm{s}^{-1}$ generated from gas moving at $\sim 70-300 \mathrm{~km} \mathrm{~s}^{-1}$. These properties are broadly consistent with the high-velocity wings observed in the SINS galaxies.

However, our spectra can also be fitted by a broad $\mathrm{H} \alpha$ line, and we therefore explore whether such a feature could likewise be generated in galactic winds. For simplicity, we assume that these winds are powered by supernova remnants (SNRs), in local examples of which broad $\mathrm{H} \alpha\left(\mathrm{FWHM}=500-8000 \mathrm{~km} \mathrm{~s}^{-1}\right)$ is observed throughout the Sedov-Taylor expansion phase, the result of charge exchange of the electrons from slow neutral atoms to the fast postshock protons (e.g., Chevalier \& Raymond 1978; Smith et al. 1991; Heng \& Sunyaev 2008). Although such broad $\mathrm{H} \alpha$ emission is not observed on the galactic scale in local starbursting systems, we note that none of these systems are appropriate analogs to the star formation mode that dominates at $z \sim 2$. Only local ULIRGs have SFR comparable to that in the SINS galaxies, and the ionized gas emission in ULIRGs suffers significantly more extinction $\left(A_{V}=5-1000\right.$; Genzel et al. 1998) than in SINS galaxies $\left(A_{V} \sim 1\right)$. We therefore briefly examine the possibility that galactic winds at $z \sim 2$ emit broad $\mathrm{H} \alpha$ lines via a superposition of SNRs.

A simple test of this scenario is whether there are a sufficient number of SNRs in the SINS galaxies to drive the observed broad $\mathrm{H} \alpha$ luminosity. Locally, SNRs are observed to have broad $\mathrm{H} \alpha$ luminosities of $10^{30}-10^{34} \mathrm{erg} \mathrm{s}^{-1}$, generated by change exchange as the shock encounters the ISM and therefore proportional to the square of the gas density. In the SINS galaxies, the high SFR $\left(\sim 100 M_{\odot} \mathrm{yr}^{-1}\right)$ and the dense ISM (1030 times denser than in local spiral galaxies) imply an increase in broad $\mathrm{H} \alpha$ luminosity per SNR by a factor of $100-1000$, to $10^{32}-10^{37} \mathrm{erg} \mathrm{s}^{-1}$. The SFR in the SINS galaxies yields $\sim 1$ supernova explosion per year, each of which will have expected Sedov-Taylor lifetimes of $\sim 10^{4} \mathrm{yr}$ in the dense ISM, resulting in roughly $10^{4}$ SNRs radiating broad $\mathrm{H} \alpha$ at any given time in the average SINS galaxy, for a total broad $\mathrm{H} \alpha$ luminosity of $10^{36}-10^{41} \mathrm{erg} \mathrm{s}^{-1}$. This number is lower than the observed broad $\mathrm{H} \alpha$ luminosity in the SINS galaxies by a factor of a few (see Table 1); however, real galactic winds penetrate much further through their host galaxies' ISM than do the sum of individual SNRs, implying that additional broad $\mathrm{H} \alpha$ emission is expected from the interaction of the large-scale winds with the ambient medium. This effect brings the predictions into even closer agreement with the observations and thus makes this mechanism an energetically feasible explanation for the luminosity and FWHM of the broad emission in the SINS galaxies.

Regardless of whether the broad emission is a broad $\mathrm{H} \alpha$ line or broad wings on all emission lines, if the emission is in fact due to starburst-driven winds, it is worth examining what the fate of this high-velocity gas may be. The average SINS galaxy (top panel of Figure 1) has either broad wings in $\mathrm{H} \alpha$ and [N II] with velocity dispersions of $\sim 250 \mathrm{~km} \mathrm{~s}^{-1}$ or a broad $\mathrm{H} \alpha$ line with velocity dispersion $\sim 700 \mathrm{~km} \mathrm{~s}^{-1}$. We can compare these values directly with the escape velocity $v_{\text {esc }} \approx$ $450 \mathrm{~km} \mathrm{~s}^{-1}$ for a typical SINS galaxy $\left(\left\langle M_{\mathrm{dyn}}\right\rangle=8 \times 10^{10} M_{\odot}\right.$, $\left\langle R_{1 / 2}\right\rangle=3.4 \mathrm{kpc}$; Förster Schreiber et al. 2009). A significant fraction ( $7 \%$ or $55 \%$, respectively) of the high-velocity gas has velocity exceeding the host galaxy's escape velocity; assuming that this gas is distributed throughout the star-forming disk, a non-negligible amount of it should be expected to escape the galaxy. Furthermore, if the surrounding dark matter halo has a flat rotation curve, this gas would also be expected to escape the halo into the intergalactic medium.

We can then estimate the mass outflow rate that would correspond to such superwinds. The escaping $\mathrm{H} \alpha$-emitting gas is $\sim 3 \%-17 \%$ of the total $\mathrm{H} \alpha$-emitting gas in the SINS galaxies (i.e., $30 \%$ of the emission is broad and $55 \%$ of the broad emission escapes). Assuming that the fraction of gas in the ionized phase is roughly the same in the galaxies' star-forming disks and in the outflows (compare $\sim 1 \%$ in the Milky Way to $\sim 0.1 \%$ $1 \%$ in winds; Veilleux et al. 2005), this implies that $\sim 3 \%$ $17 \%$ of the galaxies' gas reservoirs are being expelled by the observed star-forming event. The dynamical times associated with these outflows can be approximated as the radius of the star formation event divided by the velocity of the flow; for the SINS galaxies, this yields dynamical times of $\sim 10 \mathrm{Myr}$. The average SINS galaxy has a dynamical mass of $8 \times 10^{10} M_{\odot}$ and a gas fraction of 0.2-0.4 (e.g., Erb et al. 2006c; Bouché et al. 2007), yielding an expected outflow rate of $50-500 M_{\odot} \mathrm{yr}^{-1}$. This is 
consistent with the results of Erb (2008), who argue that the observed $z \sim 2$ mass-metallicity relationship requires outflow rates slightly larger than the SFR $\left(\mathrm{SFR}=1-800 M_{\odot} \mathrm{yr}^{-1}\right.$ with median $72 M_{\odot} \mathrm{yr}^{-1}$ in the SINS sample; Förster Schreiber et al. 2009). If the broad emission we observe is due to galactic winds at $z \sim 2$, we thus find mass outflow rates consistent with the observed metallicity evolution of these galaxies.

\subsection{Broad Emission from Active SMBHs}

Another possible, and common, interpretation of broad emission lines is as signatures of nuclear activity. For our galaxies, this interpretation is supported by the strength of the broad $\mathrm{H} \alpha$ emission increasing with stellar mass (and therefore bulge mass and possibly black hole mass) seen in Figure 2, by the lessluminous broad emission in the nonactive systems (the driving mechanism being "turned off" or obscured; Figures 1 and 2), and by the more significant detection of broad emission in the centers of galaxies than in their non-nuclear regions. Moreover, studies of $z \sim 2$ star-forming galaxies in infrared and X-ray emission suggest that active nuclei may be a common feature of this population (Daddi et al. 2007).

Early work in the local universe has shown that broad $\mathrm{H} \alpha$ is by definition omnipresent in Type $1 \mathrm{AGN}$, in which the BLRs around the nuclei are unobscured. Moreover, the kinematics and sizes of these regions can be used to infer "virial" supermassive black hole (SMBH) masses, via calibrated relations between the observed AGN continuum luminosities at $5100 \AA\left(L_{5100}\right)$ and the sizes of the BLR (Kaspi et al. 2000), which can then be used in combination with the width of the broad emission lines to estimate SMBH masses (Vestergaard 2002). Greene \& Ho (2005) have additionally related $L_{5100}$ to the luminosity of the broad emission lines, making it possible to measure virial black hole masses using only a single broad line,

$$
M_{\mathrm{BH}}=\left(2.0_{-0.3}^{+0.4}\right) \times 10^{6}\left(\frac{L_{\mathrm{H} \alpha}}{10^{42} \mathrm{erg} \mathrm{s}^{-1}}\right)^{0.55 \pm 0.02}\left(\frac{\mathrm{FWHM}_{\mathrm{H} \alpha}}{10^{3} \mathrm{~km} \mathrm{~s}^{-1}}\right)^{2.06 \pm 0.06} M_{\odot} . \text { (1) }
$$

Such virial black hole mass estimates have been verified against stellar and gas kinematic determinations of $M_{\mathrm{BH}}$ and found to be accurate to within a factor of $\sim 3$ (Onken et al. 2007; Hicks \& Malkan 2008; Netzer 2009, but see also Marconi et al. 2008). As a result, they have been utilized in the high- $z$ universe to probe the masses of SMBHs in quasars (e.g., Willott et al. 2003; McLure \& Dunlop 2004; Vestergaard 2004) and in the submillimeter-bright galaxy population (Alexander et al. 2008).

Additionally, the broad-line $\mathrm{H} \alpha$ luminosity can also be used to probe the accretion rate of SMBHs. Assuming the AGN continuum luminosity $L_{5100}$ is roughly $1 / 10$ the AGN bolometric luminosity $L_{\text {bol }}$, the accretion rate can be estimated by $\dot{M}_{\mathrm{BH}}=L_{\mathrm{bol}} /\left(\eta c^{2}\right)$ (La Mura et al. 2007). Using a typical value of $\eta \sim 0.1$ and the calibrated relationship between $L_{\mathrm{H} \alpha}$ and $L_{5100}$ (Greene \& Ho 2005), we can then estimate $\dot{M}_{\mathrm{BH}}$. Although approximate, this estimate of $\dot{M}_{\mathrm{BH}}$ and the estimate of $M_{\mathrm{BH}}$ derived using Equation (1) nevertheless allow us to study both the putative black hole masses in our SINS galaxies and their accretion rates using our measurements of the broad $\mathrm{H} \alpha$ features.

With this procedure, we derive for the average SINS spectrum (top row in Figure 1) a black hole mass of $M_{\mathrm{BH}}=4_{-2}^{+3} \times 10^{6} M_{\odot}$ with an Eddington ratio of $\sim 0.2$. Furthermore, we infer black hole masses of $M_{\mathrm{BH}}<9 \times 10^{5} M_{\odot}, M_{\mathrm{BH}}=2_{-1}^{+2} \times 10^{6} M_{\odot}$, and $M_{\mathrm{BH}}=1_{-0.5}^{+1} \times 10^{7} M_{\odot}$ in each of the three stellar mass bins, respectively. In the latter two bins, the Eddington ratios are estimated to be 0.3 and 0.4 , respectively.

We note that the $\mathrm{H} \alpha$ luminosities used in these black hole mass estimates have not been corrected for extinction, and any such correction would increase the derived masses. However, broad emission lines associated with Type 1-1.5 AGN in local galaxies are consistent with no additional extinction along the line of sight to the BLRs (e.g., Rhee \& Larkin 2000; AlonsoHerrero et al. 2003; Greene \& Ho 2005). Likewise, at $z \sim 2$, Alexander et al. (2008) have used the $\mathrm{H} \alpha / \mathrm{H} \beta$ broad-line Balmer decrement to measure only small amounts of nuclear extinction $\left(A_{V} \approx 1.2\right)$ in their dust-rich submillimeter population. These authors postulate that the plentiful dust in the submillimeter galaxies obscures regions of star formation and not the BLRs. In the SINS galaxies, the average extinction in the star-forming regions is $A_{\mathrm{H} \alpha} \sim 0.8$; if the BLR is similarly obscured, correcting for this effect would yield an increase of at most a factor of 2 in broad $\mathrm{H} \alpha$ luminosity and, from Equation (1), a factor $\sim 1.5$ in derived black hole mass. This suggests that it is unlikely that the black hole masses measured here suffer significantly from extinction.

It is then naturally interesting to compare the estimated black hole masses with large-scale galaxy properties. In the left panel of Figure 5, we find the expected trend that black holes of increasing mass are found in galaxies of increasing stellar mass, with black-hole-to-stellar mass ratios of $\sim 7 \times 10^{-5}$. For such black holes to be consistent with local scaling relations between black holes and bulges $\left(M_{\mathrm{BH}} / M_{\text {bulge }} \sim\right.$ $10^{-3}$ ), the bulge-to-total ratio $B / T$ of these systems would need to be quite small $(\lesssim 0.07)$. This is in marked contrast to results from detailed dynamical modeling of the five SINS galaxies with the highest resolution observations (Genzel et al. 2008), which yield $B / T=0.15-0.4$ (center panel of Figure 5). Parameterizing these results with a simple polynomial, we estimate "bulge" masses ( $\sim$ dynamical mass within $3 \mathrm{kpc}$ ) for the three mass bins, albeit with large uncertainty. The resulting $B / T$ for these mass bins are $<0.1,<0.4$, and 0.4 , respectively. These bulge masses are then plotted against our measured black hole masses in the right panel of Figure 5.

Comparing to the local $M_{\mathrm{BH}}-M_{\text {bulge }}$ relation measured by Häring \& Rix (2004), we find that our $z \sim 2$ galaxies lie significantly below the local relation, implying that black holes in the star-forming galaxies in the early universe may have lagged significantly behind their host bulges in assembly. Genzel et al. (2008) have shown that $z \sim 2$ is, for many of these systems, an era of bulge formation via smooth but rapid secular processes, during which massive bulges are assembled from large star-forming clumps $\left(M \sim 10^{8}-10^{9} M_{\odot}\right)$ on timescales of $\lesssim 1$ Gyr. Elmegreen et al. (2008) have demonstrated in simulations that, assuming each such clump contains a black hole of $10^{-3}$ of its total mass, these black holes would migrate to the galaxy center with their host clumps and form central SMBHs that are somewhat undermassive for the resulting bulges. The location of our $z \sim 2$ systems significantly below the local relation likewise suggests that the bulges in these galaxies form first, through rapid secular processes, with the assembly of the central SMBHs following later.

The timescale for this final SMBH growth can be estimated for the high mass bin, in which the galaxy bulges are probably largely in place at $z \sim 2$ (Genzel et al. 2008). Accretion onto the black hole at the current rate $\left(0.4 \dot{M}_{\mathrm{Edd}} \sim 5 \times 10^{-2} M_{\odot} \mathrm{yr}^{-1}\right)$ will bring these galaxies onto the local $M_{\mathrm{BH}}-M_{\text {bulge }}$ relation $\left(M_{\mathrm{BH}} \sim 10^{8} M_{\odot}\right)$ in $\sim 2$ Gyr. Similarly, galaxies in the 

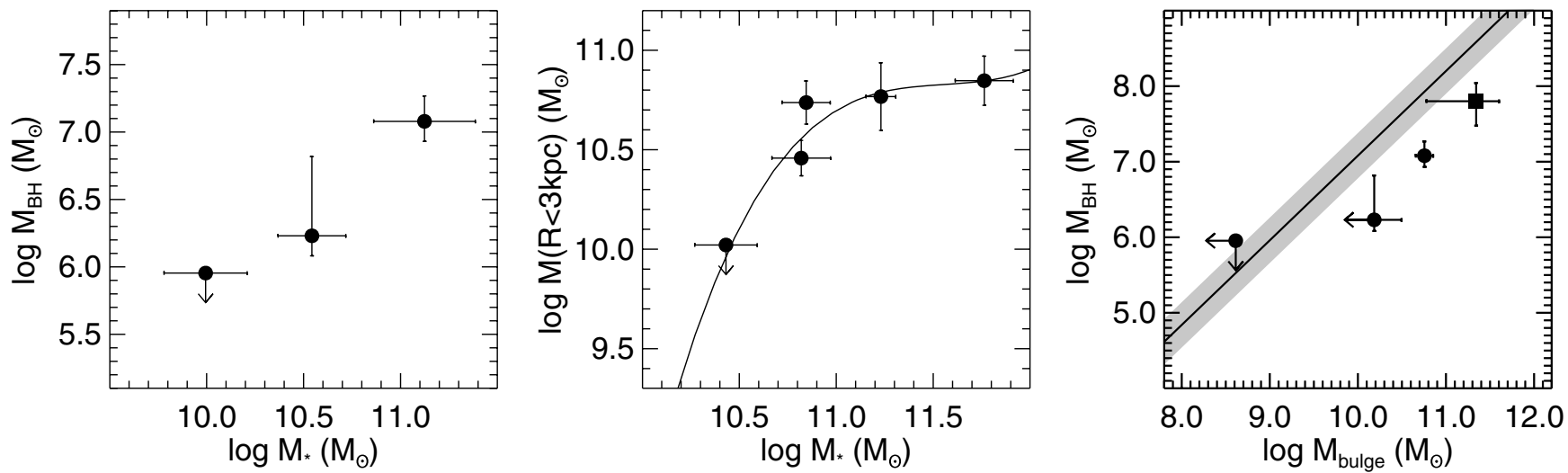

Figure 5. Left: estimated black hole masses (derived with virial estimates from the broad $\mathrm{H} \alpha$ emission line) against average stellar mass (derived with SED modeling; Förster Schreiber et al. 2009) in each mass bin. Center: relation between central mass concentration (dynamical mass within $3 \mathrm{kpc}$ ) and stellar mass for the five galaxies modeled in detail by Genzel et al. (2008), along with the best-fitting third-order polynomial. Right: estimated black hole masses against estimated bulge masses (circles; $M_{\text {bulge }}$ estimated from stellar masses shown in the left panel and polynomial shown in the center panel) for the three mass bins shown in Figure 2. Overplotted (square) is the average BH mass measured in $z \sim 2$ submillimeter galaxies by Alexander et al. (2008); for comparison with our central mass concentration (dynamical mass within $3 \mathrm{kpc}$ ), we adopt the stellar mass within $\sim 4 \mathrm{kpc}$ measured by Alexander et al. (2008) as the "bulge" mass, with the error bar extending to include all "bulge" masses estimated by those authors. The $M_{\mathrm{BH}}-M_{\text {bulge }}$ relation for the local universe and its scatter are indicated with the solid line and shading (Häring \& Rix 2004).

intermediate mass bin will acquire SMBHs with final masses $\sim 2 \times 10^{7} M_{\odot}$ in $\lesssim 1 \mathrm{Gyr}$. The evolutionary link between these final SMBHs at $z=0\left(M_{\mathrm{BH}} \gtrsim 2 \times 10^{7} M_{\odot}\right)$ and their putative $z \sim 2$ host galaxies $\left(M_{*}>10^{10} M_{\odot}\right)$ is supported by the similar space densities of these two populations $\left(\sim 3 \times 10^{-3} h_{70}^{3} \mathrm{Mpc}^{-3}\right.$ and $2 \times 10^{-3} h_{70}^{3} \mathrm{Mpc}^{-3}$, respectively; McLure \& Dunlop 2004; Daddi et al. 2004b, 2005; Reddy et al. 2005; Grazian et al. 2007). Moreover, the final black holes $\left(M_{\mathrm{BH}} \gtrsim 2 \times 10^{7} M_{\odot}\right)$ are found at $z=0$ in elliptical and bulge-dominated spiral galaxies (e.g., Tremaine et al. 2002; Marconi et al. 2004), consistent with the probable descendants of the $z \sim 2$ star-forming galaxy population (Genel et al. 2008; Conroy et al. 2008). This evidence thus confirm the plausibility of the rapid bulge formation seen at $z \sim 2$ in the SINS galaxies (Genzel et al. 2008) being followed by a few Gyr of rapid SMBH assembly, ultimately resulting in spheroids and bulges that obey the local $M_{\mathrm{BH}}-M_{\text {bulge }}$ relation.

Alexander et al. (2008) found similar delayed SMBH formation in the submillimeter galaxy population at $z \sim 2$ (see Figure 5), suggesting that the time lag between black holes and bulges may be a common phenomenon in rapidly forming galaxies at high redshift. However, this trend is not universal; quasars and radio galaxies at similar redshifts are suspected to lie above local black hole scaling relations, with black holes that are overmassive for their host bulges by up to and exceeding an order of magnitude (e.g., Walter et al. 2004; Shields et al. 2006; McLure et al. 2006; Peng et al. 2006; Maiolino et al. 2007, but see also Shields et al. 2003). These systems populate the highest mass end of the black hole mass function, with black hole masses of $>10^{8}-10^{9} M_{\odot}$ already in place at $z \sim 2$. It may therefore be that black holes in these different mass/activity regimes grow in very different circumstances and consequently relate to their bulges very differently. If this is the case, the challenge is then to locate the mechanism(s) that bring these varied high-redshift formation processes together into the black hole scaling relations observed at $z=0$.

\section{CONCLUSIONS}

In stacked, average spectra of SINS $z \sim 2$ star-forming galaxies, we have detected broad emission underneath the much brighter narrow $\mathrm{H} \alpha$ and $[\mathrm{N}$ II] emission lines. This broad emission accounts for $\sim 30 \%$ of the total $\mathrm{H} \alpha$ luminosity of these galaxies and can be parameterized equally well with a single broad $\mathrm{H} \alpha$ line ("broad line" of FWHM $\sim 1500 \mathrm{~km} \mathrm{~s}^{-1}$ ) and with a two Gaussian fitted to both the permitted and forbidden lines ("broad wings" of FWHM $\sim 550 \mathrm{~km} \mathrm{~s}^{-1}$ ). The luminosity and FWHM of the broad component increases with increasing galaxy mass and therefore with SFR. This broad component is found both in known AGNs and in stacked spectra of systems that have not been previously identified as AGNs. There is some evidence that the broad emission is more luminous in galaxy centers, as opposed to in the outer regions, but the significance of these detections are low.

We cannot empirically determine whether this broad emission is due to high-velocity galactic winds and the associated shocks or to the BLR emission of AGN. In the former case, we find that simple scaling arguments show that the luminosity and FWHM of the broad emission can plausibly be accounted for via shocking of the ambient interstellar media from supernovaedriven galactic winds. These winds would then be ejecting matter from the host galaxy at rates slightly exceeding the SFR, in keeping with expectations from the metallicity evolution of these galaxies and with ultraviolet interstellar absorption-line studies at similar redshifts.

On the other hand, the broad emission may be generated in a BLR; in this case, we can estimate the black hole masses and luminosities necessary to fuel the observed emission for each of the three galaxy mass bins. We find that the measured SMBH masses correlate with the host galaxy masses, as expected from local scaling relations, but that the SMBHs are significantly undermassive for their bulges when compared with local relations. While this result has large uncertainties, it is consistent with the emerging picture of galaxy assembly at $z \sim 2$, in which a gas-rich disk fragments into large $(\geqslant 1 \mathrm{kpc})$ superstar-forming clumps that then migrate into the galaxy center on Gyr-timescales to form a nascent bulge. The bulge would then form first through this process and only later completely assemble its black hole.

The obvious direction for future research is to determine the source of the broad $\mathrm{H} \alpha$ emission in high-redshift star-forming galaxies. This will most likely require detailed examination of individual galaxies. Among the diagnostics that will be useful for this task are comparisons with X-ray data and deep 
integrations in rest-frame UV/optical wavebands to spatially resolve, e.g., UV interstellar absorption lines and broad Balmer emission.

We thank the ESO staff, especially those at Paranal Observatory, for their helpful and enthusiastic support during the many observing runs and several years over which the SINS project was carried out. We also acknowledge the SINFONI and PARSEC teams, whose hard work on the instrument and laser paved the way for the success of the SINS observations. This paper has additionally benefited significantly from many enlightening conversations with colleagues, including Frédéric Bournaud, Mohan Ganeshalingam, Kevin Heng, Phil Hopkins, Chris McKee, Jeffrey Silverman, and Thea Steele. Finally, we thank the referee, whose detailed and insightful comments greatly improved the quality of this paper.

\section{REFERENCES}

Abraham, R. G., et al. 2004, AJ, 127, 2455

Alexander, D. M., et al. 2008, AJ, 135, 1968

Alonso-Herrero, A., Quillen, A. C., Rieke, G. H., Ivanov, V. D., \& Efstathiou, A. $2003, \mathrm{AJ}, 126,81$

Armus, L., Heckman, T. M., \& Miley, G. K. 1989, ApJ, 347, 727

Armus, L., Heckman, T. M., \& Miley, G. K. 1990, ApJ, 364, 471

Arribas, S., Colina, L., \& Clements, D. 2001, ApJ, 560, 160

Bonnet, H., et al. 2004, Messenger, 117, 17

Bouché, N., et al. 2007, ApJ, 671, 303

Chapman, S. C., Blain, A. W., Smail, I., \& Ivison, R. J. 2005, ApJ, 622, 772

Chevalier, R. A., \& Raymond, J. C. 1978, ApJ, 225, L27

Conroy, C., Shapley, A. E., Tinker, J. L., Santos, M. R., \& Lemson, G. 2008, ApJ, 679,1192

Cresci, G., et al. 2009, ApJ, 697, 115

Daddi, E., Cimatti, A., Renzini, A., Fontana, A., Mignoli, M., Pozzetti, L., Tozzi, P., \& Zamorani, G. 2004a, ApJ, 617, 746

Daddi, E., et al. 2004b, ApJ, 600, L127

Daddi, E., et al. 2005, ApJ, 626, 680

Daddi, E., et al. 2007, ApJ, 670, 173

Dickinson, M., Papovich, C., Ferguson, H. C., \& Budavári, T. 2003, ApJ, 587, 25

Eisenhauer, F., et al. 2003, Proc. SPIE, 4841, 1548

Elmegreen, B. G., Bournaud, F., \& Elmegreen, D. M. 2008, ApJ, 684, 829

Erb, D. K. 2008, ApJ, 674, 151

Erb, D. K., Shapley, A. E., Pettini, M., Steidel, C. C., Reddy, N. A., \& Adelberger, K. L. 2006a, ApJ, 644, 813

Erb, D. K., Steidel, C. C., Shapley, A. E., Pettini, M., Reddy, N. A., \& Adelberger, K. L. 2006b, ApJ, 647, 128

Erb, D. K., Steidel, C. C., Shapley, A. E., Pettini, M., Reddy, N. A., \& Adelberger, K. L. 2006c, ApJ, 646, 107

Fan, X., et al. 2001, AJ, 121, 54

Förster Schreiber, N. M., et al. 2006, ApJ, 645, 1062

Förster Schreiber, N. M., et al. 2009, arXiv:0903.1872

Genel, S., et al. 2008, ApJ, 688, 789

Genzel, R., et al. 1998, ApJ, 498, 579

Genzel, R., et al. 2006, Nature, 442, 786

Genzel, R., et al. 2008, ApJ, 687, 59

Grazian, A., et al. 2007, A\&A, 465, 393

Greene, J. E., \& Ho, L. C. 2005, ApJ, 630, 122

Häring, N., \& Rix, H.-W. 2004, ApJ, 604, L89

Heckman, T. M., Lehnert, M. D., \& Armus, L. 1993, in Astrophysics and Space Science Library Vol. 188, The Environment and Evolution of Galaxies, ed. J. Michael Shull \& H. A. Thronson (Dordrecht: Kluwer), 455

Heng, K., \& Sunyaev, R. A. 2008, A\&A, 481, 117

Hicks, E. K. S., \& Malkan, M. A. 2008, ApJS, 174, 31

Kaspi, S., Smith, P. S., Netzer, H., Maoz, D., Jannuzi, B. T., \& Giveon, U. 2000, ApJ, 533, 631
Kong, X., et al. 2006, ApJ, 638, 72

Kriek, M. 2008, ApJ, 677, 219

La Mura, G., Popović, L. C.., Ciroi, S., Rafanelli, P., \& Ilić, D. 2007, ApJ, 671, 104

Law, D. R., Steidel, C. C., Erb, D. K., Larkin, J. E., Pettini, M., Shapley, A. E., \& Wright, S. A. 2007, ApJ, 669, 929

Law, D. R., Steidel, C. C., Erb, D. K., Larkin, J. E., Pettini, M., Shapley, A. E., \& Wright, S. A. 2009, ApJ, 697, 205

Lilly, S. J., et al. 2007, ApJS, 172, 70

Lehnert, M. D., \& Heckman, T. M. 1996, ApJ, 462, 651

Maiolino, R., et al. 2007, A\&A, 472, L33

Marconi, A., Risaliti, G., Gilli, R., Hunt, L. K., Maiolino, R., \& Salvati, M. 2004, MNRAS, 351, 169

Marconi, A., Axon, D. J., Maiolino, R., Nagao, T., Pastorini, G., Pietrini, P., Robinson, A., \& Torricelli, G. 2008, ApJ, 678, 693

Martin, C. L. 1999, ApJ, 513, 156

Martin, C. L. 2003, RevMexAA Conf. Ser., 17, 56

Martin, C. L. 2005, ApJ, 621, 227

McLure, R. J., \& Dunlop, J. S. 2004, MNRAS, 352, 1390

McLure, R. J., Jarvis, M. J., Targett, T. A., Dunlop, J. S., \& Best, P. N. 2006, MNRAS, 368, 1395

Netzer, H. 2009, ApJ, 695, 793

Noeske, K. G., et al. 2007, ApJ, 660, L43

Onken, C. A., et al. 2007, ApJ, 670, 105

Peng, C. Y., Impey, C. D., Rix, H.-W., Kochanek, C. S., Keeton, C. R., Falco, E. E., Lehár, J., \& McLeod, B. A. 2006, ApJ, 649, 616

Pettini, M., Steidel, C. C., Adelberger, K. L., Dickinson, M., \& Giavalisco, M. 2000, ApJ, 528, 96

Pettini, M., Shapley, A. E., Steidel, C. C., Cuby, J.-G., Dickinson, M., Moorwood, A. F. M., Adelberger, K. L., \& Giavalisco, M. 2001, ApJ, 554 981

Puech, M., Hammer, F., Flores, H., Östlin, G., \& Marquart, T. 2006, A\&A, 455, 119

Reddy, N. A., Erb, D. K., Steidel, C. C., Shapley, A. E., Adelberger, K. L., \& Pettini, M. 2005, ApJ, 633, 748

Rhee, J. H., \& Larkin, J. E. 2000, ApJ, 538, 98

Rudnick, G., et al. 2003, ApJ, 599, 847

Rudnick, G., et al. 2006, ApJ, 650, 624

Rupke, D. S., Veilleux, S., \& Sanders, D. B. 2005, ApJS, 160, 115

Shapiro, K. L., et al. 2008, ApJ, 682, 231

Shapley, A. E., Steidel, C. C., Pettini, M., \& Adelberger, K. L. 2003, ApJ, 588, 65

Shields, G. A., Gebhardt, K., Salviander, S., Wills, B. J., Xie, B., Brotherton, M. S., Yuan, J., \& Dietrich, M. 2003, ApJ, 583, 124

Shields, G. A., Menezes, K. L., Massart, C. A., \& Vanden Bout, P. 2006, ApJ, 641,683

Smail, I., Chapman, S. C., Ivison, R. J., Blain, A. W., Takata, T., Heckman, T. M., Dunlop, J. S., \& Sekiguchi, K. 2003, MNRAS, 342, 1185

Smith, R. C., Kirshner, R. P., Blair, W. P., \& Winkler, P. F. 1991, ApJ, 375, 652

Swinbank, A. M., Smail, I., Chapman, S. C., Blain, A. W., Ivison, R. J., \& Keel, W. C. 2004, ApJ, 617, 64

Swinbank, A. M., Chapman, S. C., Smail, I., Lindner, C., Borys, C., Blain, A. W., Ivison, R. J., \& Lewis, G. F. 2006, MNRAS, 371, 465

Takata, T., Sekiguchi, K., Smail, I., Chapman, S. C., Geach, J. E., Swinbank, A. M., Blain, A., \& Ivison, R. J. 2006, ApJ, 651, 713

Tremaine, S., et al. 2002, ApJ, 574, 740

Tremonti, C. A., Moustakas, J., \& Diamond-Stanic, A. M. 2007, ApJ, 663, L77 Trujillo, I., et al. 2006, ApJ, 650, 18

Veilleux, S., Cecil, G., \& Bland-Hawthorn, J. 2005, ARA\&A, 43, 769

Vestergaard, M. 2002, ApJ, 571, 733

Vestergaard, M. 2004, ApJ, 601, 676

Walter, F., Carilli, C., Bertoldi, F., Menten, K., Cox, P., Lo, K. Y., Fan, X., \& Strauss, M. A. 2004, ApJ, 615, L17

Weiner, B. J., et al. 2009, ApJ, 692, 187

Westmoquette, M. S., Exter, K. M., Smith, L. J., \& Gallagher, J. S. 2007a, MNRAS, 381, 894

Westmoquette, M. S., Smith, L. J., Gallagher, III, J. S., O'Connell, R. W., Rosario, D. J., \& de Grijs, R. 2007b, ApJ, 671, 358

Willott, C. J., McLure, R. J., \& Jarvis, M. J. 2003, ApJ, 587, L15

Wright, S. A., et al. 2007, ApJ, 658, 78 